\title{
Emerging Trends of Ubiquitous Computing
}

\author{
Prakriti Trivedi ${ }^{1}$, Kamal Kishore Sagar ${ }^{2}$, Vernon ${ }^{3}$ \\ ${ }^{1} \mathrm{HOD}$ of CE \&IT Department \\ ${ }^{1,2,3}$ Govt. Engineering College, Ajmer \\ Rajasthan Technical University, Kota, India \\ ${ }^{1}$ hod_ceit@rediffmail.com \\ ${ }^{2}$ kamal_sa1234@yahoo.co.in \\ 3vernon86@rediffmail.com
}

\begin{abstract}
Ubiquitous computing is a method of enhancing computer use by making many computers available throughout the physical environment, but making them effectively invisible to the user. Background network to support ubiquitous computing is ubiquitous network by which users can enjoy network services whenever and wherever they want (home, office, outdoors). In this paper Issues related to ubiquitous network, smart objects and wide area ubiquitous Networks have been discussed. We also discuss various elements used in ubiquitous computing with the challenges in this computing environment
\end{abstract}

Keywords - Ubiquitous, sensor, network, wireless, computing

\section{INTRODUCTION}

Ubiquitous Computing has potential applications in several sectors such as healthcare, Business processes, disaster management, farmland irrigation and empowering the common Man to improve the quality of life. Ubiquitous computing is a method of enhancing Computer use by making many computers available throughout the physical environment, But making them effectively invisible to the user. Disappearing computer means the Functionalities of computers will be moved to the surroundings. As technology become more embedded and invisible, it calms our lives by removing the annoyances. Ubiquitous Communication is based on the concept of ubiquitous computing, where technology recedes into background of our lives to make human computer interaction much easier. They require efficient, multimedia and power-aware technologies linking together many Heterogeneous devices distributed over small or large specifications and pear-to-pear and ad hoc paradigms. Ubiquitous communications are intended to connect and transmit/distribute the information among number of computing devices that form a ubiquitous network. In ubiquitous computing the new computing devices usually equipped with a selection of different sensors to collect data from their environment. The goal is to create context awareness, which allows intelligent things to decide and act on a decentralized basis. The new computing devices are mobile, and the tasks they are programmed to perform depend on the geographical location and neighbourhood of the devices. Ubiquitous computing means anywhere (at work, at home, in the city, in the country or on move), anytime (24 hours, day or night), anyone (adults or children, elderly or

Handicapped), any thing (home appliances, individual items, cars, food products). It needs numerous base stations (BS) in cellular system and access point (AP) in wireless local area network (WLAN), which cost more. Ad- hoc networking is expected to be one of the key technologies supporting a future ubiquitous network society. Ad hoc network consists of mobile nodes equipped with wireless transceiver, which aims to establish communication anywhere anytime without the aid of infrastructure like BS and APs. Ubiquitous network allows users to exchange information through the use of broadband and mobile access. Smart objects and RFID tags are connected through wide area ubiquitous network.

\section{VARIOUS TERMINOLOGIES}

\section{A. Ubiquitous Network}

Ubiquitous network is a federation of networks on which user-oriented services are provided anywhere and anytime to a target user in the most appropriate way with null operational cost. Ubiquitous network allows all users to access and exchange information of any kind freely at anytime, from anywhere, and from any appliance through the use of broadband and mobile access as well as intelligent home appliances and RFID tags that can access networks [4]. In ubiquitous network small computers will be embedded into wearable terminals such as clothes, watches, glasses, rings, and into every object such as desks and chairs, cupboards, refrigerators, and cars. These chips and devices will be connected to each other through wireless networks and linked to the Internet. A ubiquitous network connects lots of physical objects that exist isolated in the environment [5]. It is similar to Internet that connects computers that are far apart. In order to realize the ubiquitous networks, the combination of IP network and broadband wireless access will play an important role. Ubiquitous network combines optical communication, mobile and consumer electronics into one network [1]

\section{B. Smart Objects}


Progress in technologies for sensors (to sense environment), together with the expected increase in processing power and memory, will make every object "smart". In future, more than $50 \%$ of the devices connected to the Internet will not be PCs but they will be smart devices and appliances. Smart devices are optimized to particular tasks that they blend into the world and require little technical knowledge on the part of their uses. They should be as simple as to use calculators, telephone or toasters. Networked embedded processors which forms the heart of all smart devices, will become an important R\&D field. Reliability is crucial in embedded computing systems since large interconnected information systems become unstable. The building block of an individual smart object can be derived from those of classic computer namely memory and processing, sensors and actuators as in/out devices, wireless communication to connect smart objects, architecture (hardware and software components and the interface between them) and middleware (defines how different participants communicate with one another in a network). The interaction of a person with these networks of smart things requires novel human-computer interface (HCI) i.e. special in/out devices, which enables a natural, almost unconscious interaction

\section{RF Identification}

One of the major problems in ubiquitous computing is the identification of the objects. RFID tags represent a newer and more interesting concept for identifying objects. A smart device is a small, low power microchip combined with antenna, implanted in a physical object. Each device has a unique serial number and can contain other programmable information. The information contained in a device can be transmitted to a nearby reader device by a RF signal. By holding RFID card near computing device, the reconfigured as your own. Regardless of type of computing device anything, anywhere can be configured as your own computing device.

\section{Wireless Technology}

In wireless technology Wi-Fi (Wireless Fidelity) corresponds to IEEE802.11a/b/g/n WLAN, WiMAX (Worldwide interoperability microwave access) to IEEE802.16 wireless metropolitan area network (WMAN), and Bluetooth, ultra wide band (UWB), ZigBee to IEEE802.15 wireless personal area network (WPAN). Wireless LAN operating at $2.4 \mathrm{GHz}$ can provide high speed data rates for short distances while cellular system operating at $900 \mathrm{MHz}$ covers a wide area with relatively low speed data rates. WLAN 802.11n is scheduled to be completed in June 2009 can provide data rate above $100 \mathrm{Mb} / \mathrm{s}$ in $40 \mathrm{MHz} \mathrm{BW}$ operating at $2.4 \mathrm{GHz}$. The new regulations released $255 \mathrm{Mb} / \mathrm{s}$ data rate in $5 \mathrm{GHz}$ band for indoor/outdoor use. WiMAX originally developed for fixed wireless access but later on applied to mobile wireless access (IEEE802.16e).

\section{E. Wide Area Ubiquitous Network (WAUN)}

WAUN communicate small amount of information (several Kbytes per month per device) over a wide area ( $5 \mathrm{kms}$ radius) using VHF/UHF bands [3]. Application of WAUN could be remote management, gas/water/power meter reading, health care, child tracking, stolen property (car) tracking, vending machine monitoring, environment monitoring, and community security [2]. There can be billions of objects such as mobile nodes, various services, computers, home appliances etc. connected to ubiquitous network. Taking into account the recent advances in wireless technologies such as adaptive modulation and coding (AMC), diversity antennas, and large scale monolithic microwave IC (MMIC) using CMOS devices, WAUN can be made more efficient to cover large range with high bit rate wireless link.

\section{ELEMENTS OF UBIQUITOUS COMPUTING}

Elements in ubiquitous computing mainly categorized into three types .First one is devices Second one regarding the communication link last one according to the Interfacing technology

\section{Devices}

1. Computing Nodes / Sensor-Compute Nodes (miniature to large, single to multi-core)

2. Display devices (hard and soft surface display devices)

3. Input devices (voice, video, touchpad, keypad etc.)

4. Storage devices (short-term to long-term, slow to very fast) Communication devices (wireless and wire line

\section{Communication Links}

1. Physical Links:

- Fixed wire line links

- Fixed wireless links

- Mobile wireless links

- Hybrid multi-links

2. Logical / Virtual links

\section{Interfacing technologies}

1. Navigation technologies

2. On-screen / Touch-panel technologies

3. Voice interfacing technologies

4. Video-interfacing technologies

5. Handwriting-based interfacing technologies

6. Hybrid interfacing technologies

\section{Challenges Of Ubiquitous COMPuting}

As with most new technological advances, obstacles are encountered and must be overcome for them to succeed. The idea driving ubiquitous computing is to make computers that are unseen by their users because the devices are so embedded, so natural, so ergonomic, so friendly, and so fitting, they use them without even noticing. The name alone implies computers everywhere, but in order for success to be achieved, they must dissolve into the background. To do this, ubiquitous computing devices must overcome six challenges [4] [5].

\section{A. The "Accidentally" Smart Environment -}

If you walk into an environment anywhere in the world, you would probably not find an infrastructure suitable for ubiquitous computing devices. Instead, you would find an 
infrastructure suited towards established and well-grounded technologies, such as electricity running through the walls, phone lines running into and out of buildings, and conveniences such as indoor plumbing. You are not likely to see newly constructed buildings equipped with devices to support pervasive computing.

\section{B. Improper Interoperability -}

It is common knowledge many technology-producing companies desire to produce their own proprietary products speaking their own proprietary language. This leads to "no interoperability" between devices from different companies, stunting the growth of ubiquitous computing. Even if companies agreed on a communication standard to further the new computing age, we still may see stunted growth. Even if two devices are able to communicate, there is still the question of "How well they communicate?"

\section{No Systems Administrator -}

Challenge Two above ties in nicely with the next test for ubiquitous computing to take root - lack of a systems administrator. Most individuals who operate a personal computer have no intimate knowledge of how to administer a single workstation. It would be unrealistic for the manufacturers of ubiquitous computing devices to expect their users to administer a complex network consisting of multiple devices. How does ubiquitous computing respond to this challenge?

\section{Social Implications of Aware Technologies -}

Ubiquitous computing will have a social impact on our society just as the previous two eras of computing did - there is no question about that. However, as these devices are constantly bombarded with input from their surroundings, the question then becomes what do the devices do with this input? How will it affect privacy? Will society turn to a social solution, legal solution, ethical solution, or technological solution to protect privacy?

\section{E. Reliability -}

If you take a look at the current age of computing and compare the reliability issue of today to what the reliability issue of tomorrow must be, the difference is staggering. Today's personal computers are, in a sense, becoming more and more reliable. However, they still have a long road ahead for them to catch up with the reliability exhibited by other well-founded technologies, such as televisions, telephones and even washing machines. These well-founded technologies have been successful, in part, due to their reliability.

\section{CONCLUSIONS}

Combining every object with identification, localization, and communication technologies will have a significant impact on private life of millions of people with decreasing cost of ubiquitous information and communication technology. The penetration of the technologies will become more widespread and no doubt a large number of business opportunities will emerge within this sector. Multiple devices from multiple vendors with different technological capabilities, equipped with varying communication technologies must cooperate in an ever-changing environment. Much progress in information science, computer science, and material science is necessary to render the vision economically feasible and to overcome current technological hurdles such as energy consumption. Progress in material science, chemistry and physics will change the appearance of information appliances, e.g. light emitting polymer (LEP) display, offers flexible large area or curved displays capable of delivering high resolution video images at low power consumption, visible in daylight and with wide viewing angle.

\section{REFERENCES}

[1] P. Tandler, et al, "Room ware-Moving towards ubiquitous computers", IEEE Micro, Nov.Dec, 2002, pp 36-47

[2] R. Want et al, "Disappearing hardware", IEEE Pervasive Computing, Jan-March, 2002, pp 36-47

[3] T. Starner, "The challenges of wearable computing: part 1 \&2”, IEEE Micro, July- Aug. 2001, pp 44-52 \& 54-67

[4].Tandler, P.: Software Infrastructure for a UbiquitousComputing Environment Supporting Collaboration with Multiple Single- and Multi-User Devices. Proceedings of UbiComp'01. Lecture Notes in Computer Science, Springer, Heidelberg, 96-115, 2001.

[5].Langheinrich, M. Privacy by Design - Principles of Privacy-Aware Ubiquitous Systems. in International Symposium on Ubiquitous Computing. 2001. Atlanta, USA. p. 273-291.

\section{AUTHOR PROFILE}

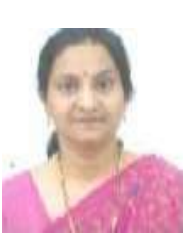

Prakriti Trivedi received her B.E. degree from MBM Engineering College,Jodhpur Rajasthan affiliated to Jai Narain University, India in 1994 and ME from NITTTR ,Punjab University ,Chandigarh , India in Computer Science \& Engineering, She is currently head of the department of CE \& IT in the Govt. Engineering College, Ajmer Rajasthan, India.

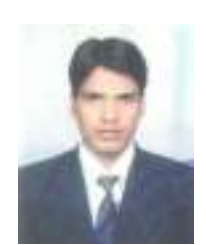

Kamal Kishore Sagar received his B.E. degree from Rajasthan University, Jaipur and currently he is pursuing M.Tech specializing in Software Engineering from Govt. Engineering College, Ajmer affilated to Rajasthan Technical University, Kota. His field of reasearch are Adhoc Networks, Security and Software engineering.

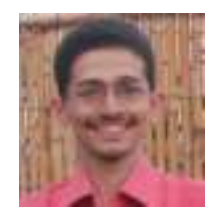

Vernon received his B.E. degree from Ajmer Institute of Technology affiliated to Rajathan University and currently he is pursuing M.Tech specializing in IT from Govt. Engineering College, Ajmer affilated to Rajasthan Technical University, Kota. His field of reasearch are Artifical Intelligence and Wireless communication technologies. 